\title{
Alternative Representations of Aberrations of the Eye.
}

Stanley A. Klein

School of Optometry

University of California

Berkeley, CA 94720

(510) 643-8670, 643-5109 (fax),

klein@ spectacle.berkeley.edu,

Daniel D. Garcia

Computer Science Division

University of California

Berkeley, CA 94720

(510) 642-9716

ddgarcia@cs.berkeley.edu.

\begin{abstract}
.
Five methods for representing pupil plane aberrations are discussed. The reference axis and reference surface can strongly affect the representation. The line of sight (an axis normal to wavefront) may not be optimal.
\end{abstract}




\title{
Alternative Representations of Aberrations of the Eye
}

\author{
Stanley A. Klein*, Daniel D. Garcia\#, University of California, Berkeley, CA 94720-2020 \\ *School of Optometry, \#Computer Science Division. (510) 643-8670, 643-5109 (fax) \\ klein@spectacle.berkeley.edu, ddgarcia@cs.berkeley.edu
}

Introduction. In recent years there has been an explosion of activity measuring the aberrations of the eye. One of the goals of this activity is to develop methods for predicting visual performance. It is not yet clear how to do that prediction. It is likely that different aspects of visual performance will require different ways of viewing the aberrations. This paper examines and compares five graphical representations of the aberration data. The advantages and disadvantages of the different displays will be discussed.

There are two domains for specifying the aberrations: the retinal plane and the pupil plane. In the retinal plane the aberrations are represented by the point spread function and its Fourier transform, the modulation transfer function. These retinal representations are sufficiently familiar that they will not be examined in the present paper. Rather, we will examine pupil plane representations of aberrations. Pupil plane aberrations have special relevance to the task of correcting the aberrations using deformable mirrors, refractive surgery and contact lenses. The most common pupil plane representation is the wavefront height. This paper discusses and compares three additional representations: the pupillary point spread function, wavefront slope and wavefront curvature.

This paper also discusses choices of the reference axis and reference surface for measuring and displaying aberrations. We will examine how the appearance of the aberrations in different representations depends on the choices made for the reference axis and reference surface.

The wavefronts to be analyzed. Two examples of aberrations will be examined. The first example will be the wavefront aberration found and plotted by Walsh, Howland \& Howland (1984) using the objective aberroscope for their subject PD. It is specified by the following Taylor expansion:

$$
\begin{aligned}
\mathrm{W}(\mathrm{x}, \mathrm{y})= & -.0033 \mathrm{x}^{3}-.0279 \mathrm{x}^{2} \mathrm{y}-.0454 \mathrm{x} \mathrm{y}^{2}+.0138 \mathrm{y}^{3} \\
& +.0141 \mathrm{x}^{4}+.0121 \mathrm{x}^{3} \mathrm{y}+.0304 \mathrm{x}^{2} \mathrm{y}^{2}+.0161 \mathrm{x}^{3} \mathrm{y}+.0126 \mathrm{y}^{4}
\end{aligned}
$$

Distances $(\mathrm{x}, \mathrm{y})$ are measured in $\mathrm{mm}$ and the wavefront, $\mathrm{W}$, is measured in microns. Walsh, et al. removed all terms below third order (the terms able to be corrected by a sphero-cylindrical lens) when they reported their data.

The second example has a wavefront given by:

$$
\mathrm{W}(\mathrm{x}, \mathrm{y})=2 \exp \left(-\left((\mathrm{x}-1)^{2}+\mathrm{y}^{2}\right) / 2\right)-2 \exp (-1 / 2) \text { microns }
$$

This wavefront is a symmetric Gaussian with a $1 \mathrm{~mm}$ standard deviation and shifted by $1 \mathrm{~mm}$ to the right. This wavefront was chosen to simulate a very mild keratoconic distortion with peak curvature of 2 diopters $(500 \mathrm{~mm}$ radius of curvature of the wavefront). A more typical keratoconic distortion would have a peak power of $20 \mathrm{D}$ rather than $2 \mathrm{D}$. By choosing an aberration of $2 \mathrm{D}$ we are in the range of aberrations of normal corneas.

Around the symmetry axis (at $\mathrm{x}=1 \mathrm{~mm}$ ) this wavefront can be expanded as:

$$
\mathrm{W}(\mathrm{x}, \mathrm{y})=2\left[\left(1-\exp (-1 / 2)-\left((\mathrm{x}-1)^{2}+\mathrm{y}^{2}\right) / 2+\left((\mathrm{x}-1)^{2}+\mathrm{y}^{2}\right)^{2} / 8\right]+\right.\text { higher order terms }
$$

The constant terms give the height of the peak of the Gaussian. The quadratic terms correspond to the spherical wave with a $500 \mathrm{~mm}$ radius of curvature. There is no cylinder term present around the symmetry axis. The fourth order terms correspond to spherical aberration. There are no cubic (coma) terms around the symmetry axis. The term $2 \exp (-1 / 2)$ is subtracted off since in all of our examples we have chosen the wavefront height to be zero at the origin $(\mathrm{x}=\mathrm{y}=0)$.

Around the original axis $(\mathrm{x}=\mathrm{y}=0)$, the wavefront of Eq. 1 can be expanded as:

$$
\begin{aligned}
\mathrm{W}(\mathrm{x}, \mathrm{y}) & =2\left[\exp \left(-\mathrm{r}^{2} / 2\right) \exp (\mathrm{x})-1\right] \exp (-1 / 2) \\
& =\mathrm{K}\left[\left(1-\mathrm{r}^{2} / 2+\mathrm{r}^{4} / 8+\ldots\right)\left(1+\mathrm{x}+\mathrm{x}^{2} / 2 !+\mathrm{x}^{3} / 3 !+\mathrm{x}^{4} / 4 !+\ldots\right)-1\right] \\
& =\mathrm{K}\left[\mathrm{x}-\mathrm{y}^{2} / 2+\left(\mathrm{x}^{3} / 3-\mathrm{x} \mathrm{r}^{2}\right) / 2+\left(\mathrm{x}^{4} / 24+\mathrm{x}^{2} \mathrm{r}^{2} / 4+\mathrm{r}^{4} / 8\right)+\ldots\right]
\end{aligned}
$$

where $\mathrm{K}=2 \exp (-1 / 2)$ and $\mathrm{r}^{2}=\mathrm{x}^{2}+\mathrm{y}^{2}$.

We can identify the various terms as follows:

paraxial spherical aberration: $\quad \mathrm{K}\left(\mathrm{x}^{4} / 24+\mathrm{x}^{2} \mathrm{r}^{2} / 4+\mathrm{r}^{4} / 8\right)$

paraxial coma:

$\begin{array}{ll}\text { paraxial sphero-cylinder: } & -\mathrm{Ky}^{2} / 2 \\ \text { paraxial prism: } & \mathrm{Kx}\end{array}$

paraxial piston (offset at origin): 0

Fig. 1 shows five different representations of aberrations (the rows of Fig. 1) for five wavefront shapes (the columns). The columns are the following shapes:

Column 1: Eq. 6 with all the terms.

Column 2: Eq. 6 minus the paraxial prism term.

Column 3: Eq. 6 minus the shifted spherical term, $\mathrm{K}\left((\mathrm{x}-1)^{2}+\mathrm{y}^{2}\right) / 2$

Column 4: Eq. 6 minus all terms less than cubic order

Column 5: Eq. 1 from the Walsh, et al. (1986) paper. 
The rationale for the various manipulations of the lower order terms in the first four columns is as follows. The first column is the original wavefront that has full symmetry around the shifted axis (at $\mathrm{x}=1 \mathrm{~mm}$ ). In the second column the removal of the prism is equivalent to a rotation around the $y$ axis. Prism is equivalent to a rotation as long as the rotation is very small. In our case a prism wavefront of $\mathrm{Kx}$ (Eq. 7d) corresponds to a wavefront slope at the origin of $\mathrm{K}=2 \exp (-1 / 2)=1.213$ milliradians $=0.1213$ prism diopters $=4.17 \mathrm{~min}$. Slope (in min.) is especially interesting since that is the basis of our point spread function representation (the gray curve in Fig. 3a shows that the slope is $4.17 \mathrm{~min}$ at the origin). The third column of Fig. 1 removes the prism by removing just the right amount of displaced sphere so no rotation is needed. Finally, the fourth column removes the remaining second-order terms, leaving only the aberrations. A similar strategy was used by Walsh, et al. (1984), shown in the fifth column.

\section{Five pupil based representations of aberrations}

1. Wavefront elevation. The first representation (Fig. 1) is the standard wavefront contour plot. Contour lines at -2 , $-1,0,2$ and 6 microns are shown as indicated by the colorbar legend on the right. In all of our examples we have chosen the piston term so that the wavefront height at the origin is zero. In our contour plots values that are more negative than the colorbar range are represented by white. Values that are more positive than the colorbar range are represented by the darkest color of the colorbar. The wavefront representation is especially relevant for refractive surgery since $\mathrm{W}(\mathrm{x}, \mathrm{y}) /(\mathrm{n}-1)$ is close to the amount of cornea to be ablated (Klein, 1998), where $\mathrm{n}$ is the corneal index of refraction (one must account for epithelial regrowth and corneal reshaping in planning the surgery).

2. Wavefront slope. The Pupillary Point Spread Function. The second representation of aberrations (Fig. 2) is new, as far as we know. We call it the pupillary point spread function (PPSF) which can be thought of as the image space retinal PSF brought to object space and referenced to the pupil plane. The PPSF is given by:

$$
\operatorname{PPSF}(\mathrm{x}, \mathrm{y})=3.438 * \operatorname{magnitude}(\operatorname{grad}(\mathrm{W}(\mathrm{x}, \mathrm{y})))
$$

where $3.438=57.3 * 60 / 1000$ converts milliradians to $\mathrm{min}$ arc, and the gradient of $\mathrm{W}$ is a vector given by:

$$
\operatorname{grad}(\mathrm{W})=(\partial \mathrm{W} / \partial \mathrm{x}, \partial \mathrm{W} / \partial \mathrm{y})=-2(\mathrm{x}-1, \mathrm{y}) \mathrm{W}(\mathrm{x}, \mathrm{y}) \text { for the Eq. } 2 \text { wavefront. }
$$

The quantity in Eq. 9 is the slope of the object space ray at pupil location (x,y) directed to a fixed retinal point (like the center of the fovea). It is precisely the slope being measured by a Hartmann-Shack sensor. Since it is close to the raw data it should be fairly independent of modeling assumptions.

The PPSF is probably the most clinically relevant of all the pupil plane representations since it directly shows the blurring in minutes of arc in object space. The lightest shading of gray shows the regions of the pupil in which the blur is less than one minute. This is the region contributing to 20/20 acuity. Figs. 1 and 2 show that the representations of aberrations depend on what is chosen as the reference axis and the reference surface. Panels a-d are representations of the same waveform, yet they look different from each other.

Figure 3 shows horizontal cuts of Figs. 1 (black) and 2 (gray) along the x axis. These plots clarify the magnitude of the aberration with the wavefront (Fig. 1) in microns and the point spread function (Fig. 2) in minutes.

3. Wavefront slope. The Tscherning "aberroscope grid". Our second representation, Eq. 8, was a contour plot of the magnitude of the wavefront slope. There are other ways to represent a slope. One could place short arrows at an array of pupil samples (Barsky, et al., 1997). The length of the arrow would be given by Eq. 8 and the direction would be the direction of the slope. Although this can be a useful representation it is often difficult to discern the direction of the arrows and the picture can get cluttered. An alternative is to connect the tips of the arrows making a grid. That is our third representation (Fig. 4). It is similar to the representation that Howland \& Howland (1976) used for their crossed-cylinder aberroscope. It is even closer to the Tscherning aberroscope (using a plus lens rather than a cross-cylinder). The main difference is that our representation is in object space whereas Tscherning's was in image space, on the retina. Our intersection points are similar to an object space spot diagram.

4 and 5. Wavefront curvature (refractive power). Figures 5 and 6 are two representations of the curvature of the wavefront. Since the wavefront slope is so small (see Fig. 2) the curvature is simply the second derivative of the wavefront. There are several different ways to specify the wavefront curvature. One begins by taking three second derivatives of the waveform: $\mathrm{W}_{\mathrm{xx}}=\partial^{2} \mathrm{~W} / \partial \mathrm{x}^{2}, \mathrm{~W}_{\mathrm{yy}}=\partial^{2} \mathrm{~W} / \partial \mathrm{y}^{2}, \mathrm{~W}_{\mathrm{xy}}=\mathrm{d}^{2} \mathrm{~W} / \partial \mathrm{x} \partial \mathrm{y}$. These second derivative are components of refractive power of the incoming waveform needed to produce a good focus.

There are quite a few ways to combine the three second derivative maps to get integrated power maps:

- $\quad$ Mean curvature, or Laplacian, or Equivalent sphere: $\mathrm{ES}=\left(\mathrm{W}_{\mathrm{xx}}+\mathrm{W}_{\mathrm{yy}}\right) / 2$

- Jackson cross cylinder at $0, \mathrm{~J}_{0}=\left(\mathrm{W}_{\mathrm{xx}}-\mathrm{W}_{\mathrm{yy}}\right) / 2$

- Jackson cross cylinder at $45, \mathrm{~J}_{45}=\mathrm{W}_{\mathrm{xy}}$

- Total Jackson cylinder, $\mathrm{C}=\operatorname{sqrt}\left(\mathrm{J}_{0}{ }^{2}+\mathrm{J}_{45}{ }^{2}\right)$

- $\quad$ Maximum and minimum curvatures $=\mathrm{ES}+\mathrm{C}$ and $\mathrm{ES}-\mathrm{C}$

- Meridional curvature. The component of curvature in meridional direction.

Several of these representations are already familiar from methods used to represent corneal topography information. Barsky, Klein \& Garcia (1997) showed a number of representations for a keratoconic cornea. They described meridional curvature (they called it instantaneous curvature) and mean curvature (very close to their Gaussian curvature). They showed how cylinder could be illustrated as short oriented lines on the mean curvature plot. For Figures 5 and 6 we have chosen to illustrate a different curvature (also called refractive power since we are discussing the refracted wavefront): maximum and minimum curvature. We have found maximum and minimum curvatures to be especially useful for displaying refractive powers after refractive surgery. 
Results. The right-hand column of Figs. 1-7 are representations of the wavefront presented in Fig. 2 of Walsh, et al. (1984). Our Figs. 1e and 4e correspond to their Figs. 2c and 2b respectively. Our inner contour line shows where the wavefront is between -1 and 0 microns $(\mu)$ and the middle zone is the region between 0 and $2 \mu$. The negative values are caused by the presence of coma. Panel $2 \mathrm{e}$ shows that there is a region with a diameter of around $4 \mathrm{~mm}$ where the amount of blur is less than 1 min. The finding that the various panels in $1 \mathrm{e}-7 \mathrm{e}$ are symmetric around the origin indicates that coma is probably fairly small for this individual. In fact, as seen in Table 3 of Walsh, et al. (1984) observer PD has the smallest amount of coma of their 11 observers. Plot 5e of maximum curvature shows there is a region with about a $4 \mathrm{~mm}$ diameter where the maximum curvature is less than 1 diopter. Plot $6 \mathrm{e}$ of minimum curvature has a central region that is negative, due to the comatic contribution. However, as seen in Fig. $7 \mathrm{e}$, this negative curvature is very close to zero.

Panels a-d are all for the same Gaussian wavefront described by Eqs. 2-8. The different columns all have identical Taylor expansion terms above second order. They differ by what is done with the direction of the reference axis (the amount of prism) and what is done with the sphero-cylindrical correction. Panels d, are the representations that are similar to what Walsh et al. (1984) did, namely remove all the Taylor expansion terms below third order. We would like to argue that this may not be the optimal way to view the aberrations or their effect on vision.

The left-hand panels (column a) represent the original displaced Gaussian wavefront. The Gaussian shape of the wavefront is most easily discerned in panel 1a. The position of the zero of the wavefront is totally arbitrary. We have chosen to place the zero at the origin. One of the most instructive pictures for assessing the expected visual quality is Fig. 2. Panel 2a shows that there is a large outer ring (the region more than $2 \mathrm{~mm}$ from the center of the Gaussian) where the blur is less than $1 \mathrm{~min}$. We would expect this individual to have quite good vision for a dilated pupil because of the large outer region with minimal blur. The gray line in Fig. 3 showing a horizontal cut through the middle of Fig. 2 may be an easier way to gain an appreciation for what the contour lines of Fig. 2 are representing. It should be noted that the spot ("spider-web") diagrams of Fig. 4 are based on the same gradient information used to construct Fig. 2. The enlargements that are seen in panels $4 \mathrm{c}$ and $4 \mathrm{~d}$ are due to the removal of sphere (in 4c) and sphero-cylinder (in 4d). The Fig. 4 representation is able to provide a very quick view (for experienced "spider-web" interpreters) of the relative amounts of the different categories of aberration.

A possible problem with column a is that the axis isn't along the line of sight, since the wavefront for column a isn't perpendicular to the axis. Three methods for making the reference axis normal to the wavefront are examined in columns b (remove the prism term), c (remove a spherical term) and d (remove all terms below cubic). Panels $1 \mathrm{~b}$, $2 \mathrm{~b}$, and $3 \mathrm{~b}$ show that the removal of prism introduces a tilt that can greatly confuse the understanding of the aberrations. Removal of an optimal amount of sphero-cylinder is probably the best approach. Further research is needed to determine the optimal sphero-cylindrical reference.

Discussion. The usual method for representing aberrations at the pupil plane is to show a contour map of the wavefront. The best fitting sphero-cylindrical correction is often subtracted out. In this paper we showed two additional representations based on the wavefront slope and two based on the wavefront curvature. The representation that gives direct insight about how the aberrations affect visual performance is the pupillary point spread function (Fig. 2). We also showed that the representation of the aberrations can depend strongly on the choice of reference axis and reference sphere. We argue that the line of sight might not be the best reference axis. Further research is needed to determine the optimal reference axis and optimal sphero-cylindrical reference surface. Just as these considerations are relevant for how to display the aberrations, they are also relevant for how much cornea to ablate in refractive surgery. The amount of cornea to be ablated will depend on what one chooses for the reference axis and what one chooses for the residual amount of sphere and cylinder. A judicious choice of these quantities can result in a residual optics with extended depth of focus with less cornea being ablated than would be the case for a "more obvious" choices of axis and residual refraction.

\section{References}

Barsky, B. A., Klein, S. A., and Garcia, D. D. (1997). "Gaussian power with cylinder vector field representation for corneal topography maps". Optom. and Vision Sci. 74, 917-925.

Howland, B. and Howland, H. C. (1976). "Subjective measurement of high-order aberrations of the eye". Science 193, 580-582.

Klein, S. A. (1998). "Optimal corneal ablation for eyes with arbitrary Hartmann-Shack aberrations". J. Opt. Soc. Am. A, 15, 2580- 2588.

Tcherning, M. (1894). "Die monochromatischen abberationen des menschlichen auges". Z. Psychol. Physiol. Sinn. 6, 456-471.

Walsh, G., Charman, W. N., and Howland, H. C. (1984). "Objective technique for the determination of monochromatic aberrations of the human eye". J. Opt. Soc. Am A, 1, 987-992. 
Figure 1 (a-e) : Wavefront (microns)
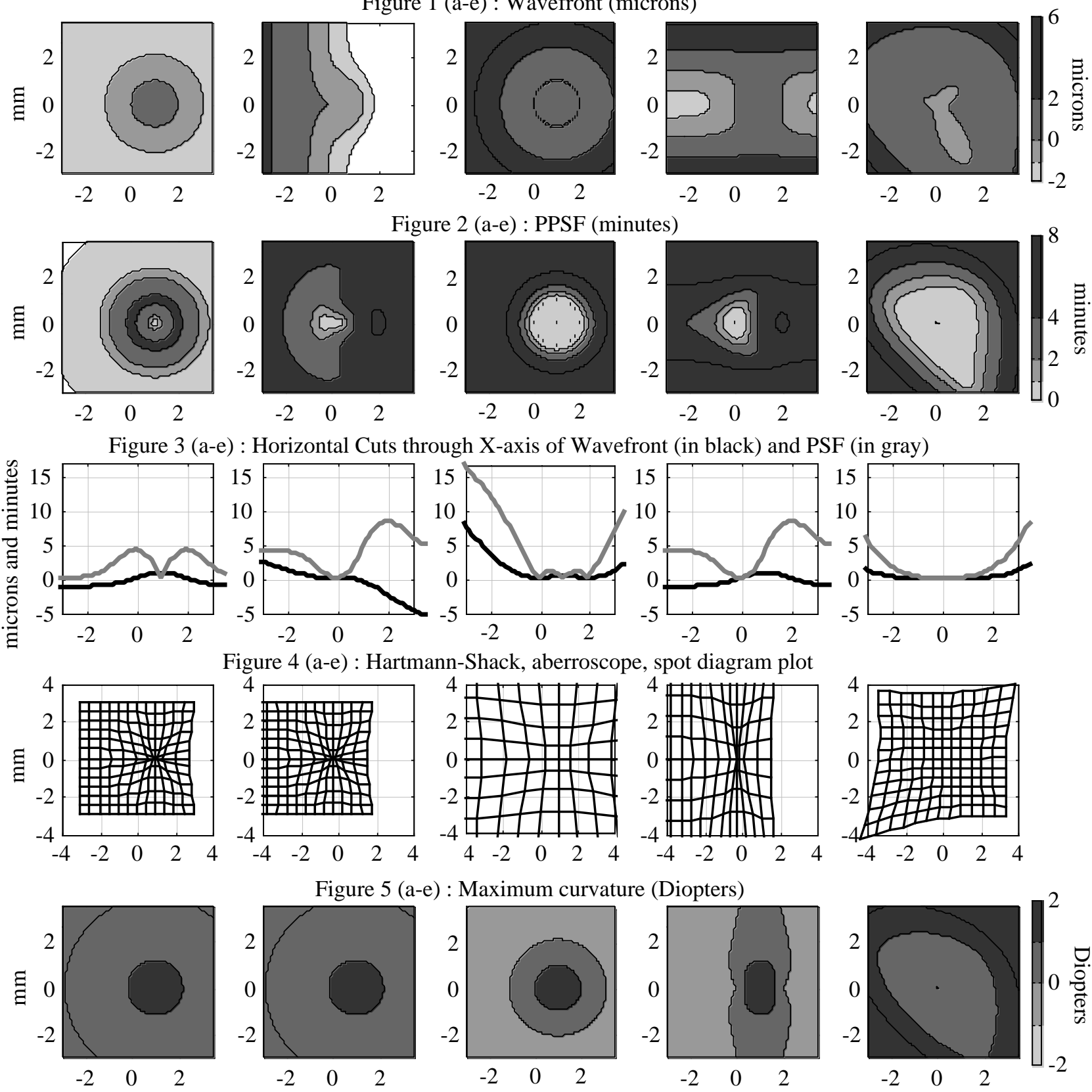

Figure 5 (a-e) : Maximum curvature (Diopters)
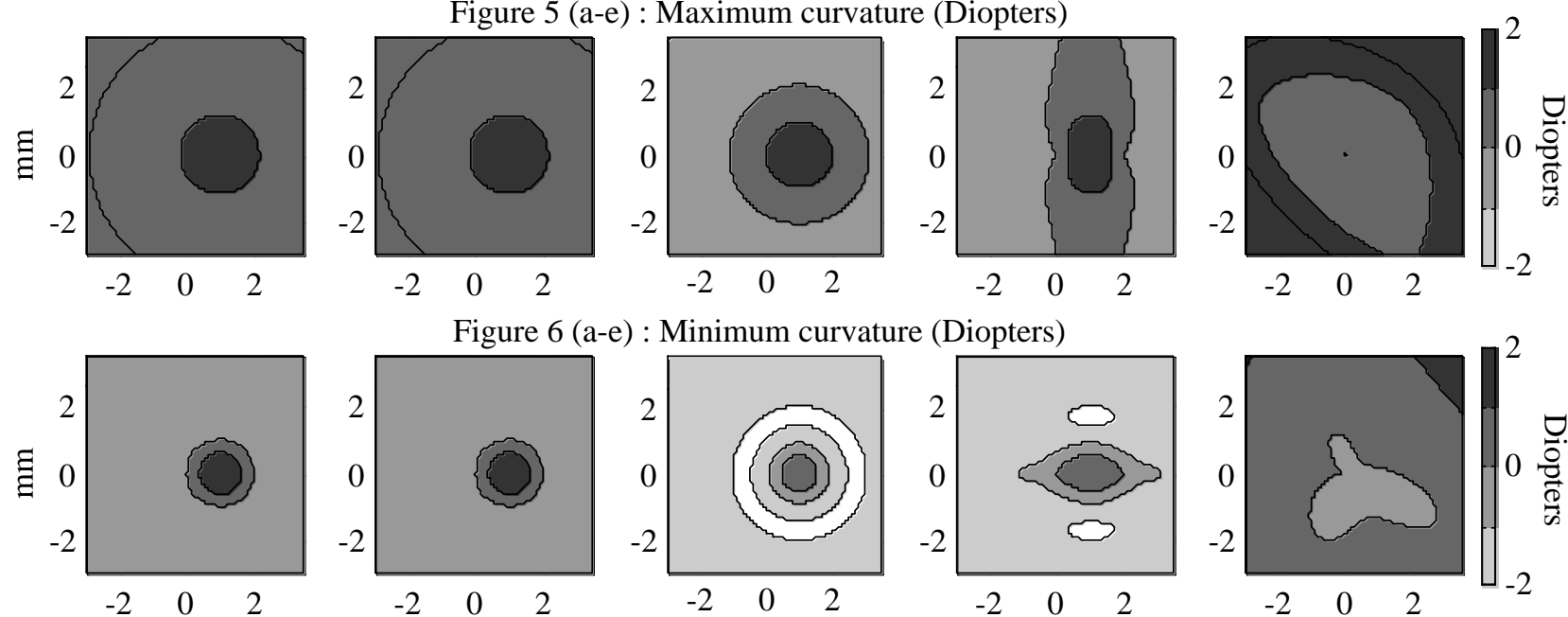

Figure 7 (a-e) : Horizontal cuts through X-axis of Maximum (in black) and Minimum curvature (in gray)
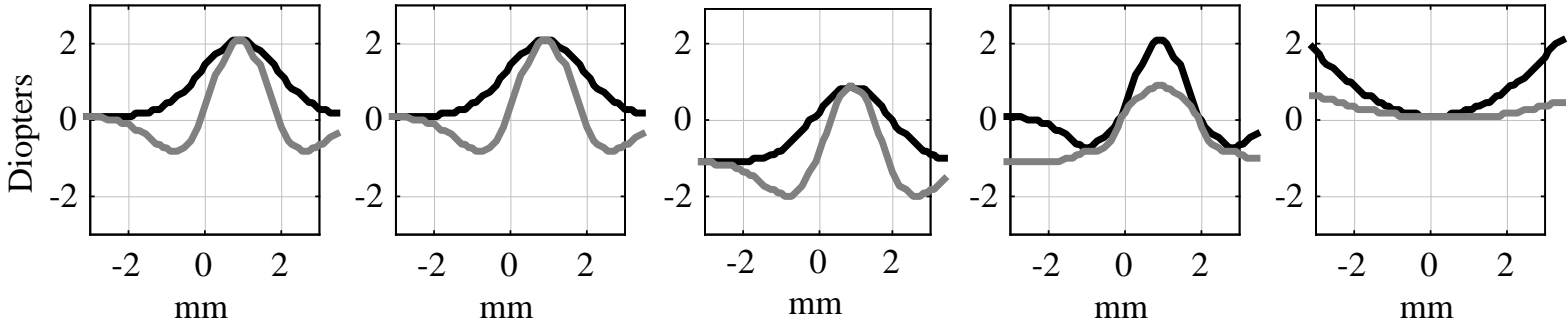